\title{
Processo de trabalho de enfermeiras na consulta ginecológica
}

\author{
Nurses work process in gynecological \\ consultation
}

\section{Leonardo Lima Ribeiro' 1 Ângela Cristina Fagundes Góes ${ }^{2}$ (1)}

${ }^{1}$ Autor principal para correspondência. Universidade do Estado da Bahia (Salvador). Bahia, Brasil. leoribeiro9565@gmail.com Universidade do Estado da Bahia (Salvador). Bahia, Brasil. angelacfgoes@gmail.com

RESUMO | OBJETIVO: Desvelar o processo de trabalho de enfermeiras na consulta ginecológica na estratégia saúde da família. MÉTODO: Trata-se de uma pesquisa descritiva, exploratória e qualitativa realizada com doze enfermeiras de Unidades Básicas de Saúde da Família de um Distrito Sanitário de um município baiano. Utilizou-se um roteiro de entrevista semiestruturado contendo perguntas abertas e fechadas. A análise dos dados foi realizada através da técnica de Análise de Conteúdo de Bardin. RESULTADOS: Após a análise e interpretação dos dados emergiram duas categorias: Abordagens sobre a consulta de enfermagem ginecológica na ESF; O processo de trabalho de enfermeiras na consulta de enfermagem ginecológica. CONCLUSÃO: A consulta ginecológica faz parte das ações integrantes do processo de trabalho das enfermeiras nas Unidades Básicas de Saúde da Família, contudo, as profissionais não visualizam o seu protagonismo neste atendimento e apresentam práticas limitadas, mostrando uma visão reducionista da assistência. Sobrecarga de trabalho, fragilidade na educação permanente e inadequações estruturais prejudicam o processo de trabalho desses profissionais e a atenção à saúde da mulher.

DESCRITORES: Consulta de enfermagem ginecológica. Atenção Primária à Saúde. Estratégia Saúde da Família. Ginecologia.
ABSTRACT | OBJECTIVE: To reveal the work process of nurses in gynecological consultation in the family health strategy. METHOD: This is descriptive, exploratory, and qualitative research carried out with twelve nurses from Basic Family Health Units in a Health District in a municipality in Bahia. A semi-structured interview script containing open and closed questions was used. Data analysis was performed using the Bardin Content Analysis technique. RESULTS: After analyzing and interpreting the data, two categories emerged: Approaches to gynecological nursing consultation in the FHS; The work process of nurses in the gynecological nursing consultation. CONCLUSION: The gynecological consultation is part of the actions that are part of the nurses' work process in the Basic Family Health Units, however, the professionals do not see their role in this service and present limited practices, showing a reductionist view of care. Work overload, fragility in permanent education, and structural inadequacies affect the work process of these professionals and the attention to women's health.

DESCRIPTORS: Gynecological nursing consultation. Primary Health Care. Family Health Strategy. Gynecology. 


\section{Introdução}

A Atenção Básica (AB) no Brasil caracteriza-se por um conjunto de ações, no âmbito individual e coletivo, que visa a promoção da saúde, prevenção de agravos, diagnóstico precoce, tratamento e reabilitação, e deve ser a principal "porta de entrada" dos usuários na Rede de Atenção à Saúde (RAS). Entre os seus princípios estão: universalidade, vínculo, responsabilização pelo cuidado e a integralidade ${ }^{1,2}$.

A Política Nacional de Atenção Básica publicada em 2017 reforça que o entendimento global deve ser priorizado, buscando a singularidade e inserção cultural das pessoas, deixando de lado concepções simplistas e mecânicas, anteriormente comuns nos atendimentos. Nesse sentido, por ter apoio multiprofissional e maior resolutividade, a Estratégia Saúde da Família (ESF) é responsável pela consolidação e, principalmente, qualificação da $A B^{3}$.

O processo de trabalho é entendido como a transformação de determinado objeto em produto ou serviço, através da ação intencional e consciente do ser humano, de modo que o produto tenha valor para o próprio ser humano. Nesse sentido, o processo de trabalho em saúde refere-se ao fazer cotidiano dos profissionais de saúde nos serviços com vistas à produção de cuidado o que implica na construção de relações interpessoais. Assim, o processo de trabalho em enfermagem tem como produto o cuidado no âmbito biológico, psicológico, social e espiritual voltado para as necessidades de indivíduos, famílias e comunidades. Logo, a produção do cuidado vai além da realização de procedimentos, pois envolve a promoção de saúde, prevenção de doenças e agravos com valorização das singularidades e da autonomia dos sujeitos envolvidos 4 .

No que diz respeito à atenção à saúde da mulher, apesar dos avanços obtidos desde a implantação do Programa de Assistência Integral à Saúde da Mulher PAISM em 1984, foi somente em 2004 com a criação da Política Nacional de Atenção Integral à Saúde da Mulher - PNAISM que houve a ampliação de possibilidades concretas de ações voltadas para este público em uma perspectiva integral. Assim, a atenção a esse grupo populacional engloba, entre outros aspectos, a assistência clínica e ginecológica em todas as fases do ciclo vital, tais como prevenção, diagnóstico e tratamento das infecções sexualmente transmissíveis (IST) e rastreamento do câncer de colo do útero e de mama 5 .
Todavia, os serviços de saúde ainda reproduzem atitudes próprias do modelo médico hegemônico focado na doença e seus desdobramentos, apoiados em tecnologias duras, de modo que, no que tange à assistência prestada à mulher na $A B$, mudar o paradigma reducionista das consultas ginecológicas não é tarefa fácil. Nesse sentido, a enfermagem entra como protagonista nesse desafio, pois na maioria das unidades de saúde tais consultas são realizadas por esses profissionais ${ }^{5-7}$.

A consulta ginecológica, além da anamnese e exame físico para diagnóstico e conduta relacionada à terapêutica, deve abordar temas relacionados à sexualidade da mulher, planejamento familiar, histórico ginecológico e obstétrico e aspectos psicológicos, estimulando o autocuidado, acolhendo e valorizando o encontro com a usuária ${ }^{8-9}$. No entanto, estudo mostra que a Consulta Ginecológica de Enfermagem na Estratégia Saúde da Família (ESF) se limita à observação da genitália e coleta citopatológica, onde os profissionais observam apenas a queixa do dia, não buscando outras informações, tais como estilo de vida e as relações sociais da usuária ${ }^{10}$.

Portanto, a consulta ginecológica constitui-se em espaço importante para o estabelecimento de diálogo e vínculo respeitoso e de confiança entre a usuária e o profissional que lhe assiste, requerendo por parte das enfermeiras e da gestão, atenção cuidadosa que a qualifique e valorize, gerando maior satisfação por parte dos profissionais e das usuárias assistidas, exigindo competências, habilidades e empenho para o enfrentamento dos desafios com vistas a assegurar um cuidado à saúde da mulher integral, longitudinal e com qualidade.

Partindo do pressuposto que a consulta ginecológica faz parte da agenda semanal de trabalho da enfermeira na ESF e que o conhecimento sobre ela é importante para a assistência à mulher em sua integralidade, essa pesquisa tem como questão norteadora: Como se dá o processo de trabalho de enfermeiras na consulta ginecológica na ESF?

A relevância desse estudo, cujo objeto é o processo de trabalho de enfermeiras (os) na consulta ginecológica na ESF, está na importância da consulta ginecológica no cuidado com a saúde da mulher, atividade das mais demandadas pelas usuárias nas Unidades de Saúde da Família do Distrito Sanitário (DS) participante do estudo. 
Ademais, com o aumento do número de consultas por turno de atendimento, para cada enfermeira, o processo de trabalho dessas profissionais se tornou mais desafiador. E, considerando o quantitativo de 10 Unidades Saúde da Família existentes no DS, com uma média de quatro enfermeiras nas equipes por unidade, a complexidade aumenta dado a necessidade de alinhamento de condutas no território no que diz respeito à consulta ginecológica, o que motivou nosso interesse por realizar essa pesquisa.

$O$ estudo se justifica pelos benefícios que pode trazer para o DS no que diz respeito à operacionalização das consultas ginecológicas de enfermagem nas suas Unidades Saúde da Família, contribuindo com reflexões para uma atenção à saúde da mulher mais resolutiva e transformadora. Neste contexto, este estudo tem como objetivo desvelar o processo de trabalho de enfermeiras na consulta ginecológica na estratégia saúde da família.

\section{Método}

Estudo descritivo, exploratório e qualitativo realizado com enfermeiras das Unidades Básicas de Saúde com Estratégia Saúde da Família de um Distrito Sanitário (DS) de um município baiano. O referido DS tem a segunda maior área de abrangência, compreendendo 17 bairros e uma população estimada em 2017 de 425.991 pessoas, sendo a maioria negra e de baixa renda.

Os participantes do estudo foram selecionados com base nos critérios de inclusão previamente estabelecidos: ser enfermeira de UBS com ESF do DS em estudo; ter tempo de atuação mínimo de seis meses na ESF; realizar consulta ginecológica na UBS; estar presente nos dias de coleta e aceitar participar da pesquisa assinando o Termo de Consentimento Livre e Esclarecido. Após a aplicação dos critérios de inclusão, a seleção dos profissionais a serem entrevistados foi de conveniência considerando a disponibilidade dos mesmos para a entrevista nos turnos em que o entrevistador estava na unidade. Todos os profissionais abordados aceitaram participar da entrevista, exceto aqueles que estavam realizando atendimentos e com pacientes em espera.
A quantidade de participantes foi definida pela saturação dos dados, segundo a relevância dos conteúdos dos discursos pertinentes ao desenvolvimento do objeto desta pesquisa, totalizando 12 Enfermeiras.

Os dados foram coletados entre os meses de junho e julho de 2019, por meio da técnica de entrevista, utilizando um roteiro semiestruturado contendo perguntas fechadas e abertas, dando ao entrevistado a possibilidade de falar sobre o assunto proposto, sem condições ou respostas pré-estabelecidas pelos pesquisadores.

O roteiro de entrevista foi organizado em duas partes, a primeira com solicitação de informações para traçar o perfil dos participantes e, a segunda parte com questões relativas ao objeto de estudo: "O que você entende por consulta ginecológica de enfermagem?”, “Em sua opinião, qual a importância da consulta ginecológica de enfermagem?", "Como são organizadas as consultas ginecológicas de enfermagem na sua equipe?", "Quais os aspectos relativos à saúde da mulher você aborda na consulta ginecológica de enfermagem?", "Quais as facilidades e dificuldades encontradas por você na USF que atua para a realização da consulta ginecológica de enfermagem?".

As entrevistas foram realizadas individualmente e em ambiente privativo, sendo gravadas mediante autorização da pessoa entrevistada, a fim de se obter o máximo de informações e assegurar o alcance dos objetivos delineados. Os conteúdos gravados foram transcritos e para preservar o anonimato, cada participante foi identificado com a letra " $E$ " de enfermeira, seguido por um número aleatório de 1 a 12 (E1 a E12), que não corresponde à ordem das entrevistas. Posteriormente os dados transcritos foram analisados com base na técnica de análise de conteúdo proposta por Bardin ${ }^{11}$, compreendendo as seguintes fases: pré-análise; exploração do material e tratamento dos resultados, inferência e interpretação.

Esta pesquisa foi aprovada pelo Comitê de Ética em Pesquisa da Universidade do Estado da Bahia (CAAE 16280919.0.0000.0057). Foram seguidas todas as recomendações necessárias para a pesquisa com seres humanos estabelecidas pela Resolução N 466 de 12 dezembro de 2012. 


\section{Resultados e discussão}

Participaram da pesquisa doze enfermeiras, sendo dez do sexo feminino; as idades variaram de 30 a 57 anos; onze tinham pelo menos uma especialização em saúde; o maior tempo de experiência em ESF foi de dezenove anos e o menor um ano e seis meses.

A partir da análise dos dados obtidos, emergiram duas categorias: Abordagens sobre a consulta de enfermagem ginecológica na ESF; O processo de trabalho de enfermeiras na consulta de enfermagem ginecológica.

\section{Abordagens sobre a consulta de enfermagem ginecológica na ESF}

A compreensão que a enfermeira tem sobre consulta de enfermagem ginecológica interfere na sua prática profissional quando realiza esse tipo de atendimento. Dessa forma, observou-se que os participantes dessa pesquisa compreendem que a consulta de enfermagem ginecológica não se limita apenas à coleta de material para exame ou à queixa da usuária, mas valorizam o olhar multidimensional:

O preventivo não se resume apenas a coleta, mas sim a consulta de enfermagem. (E4)

Não é só queixa-conduta, mas saber as dúvidas [...]. (E2)

Quando eu atendo uma paciente eu atendo de forma holística [...]. (E5)

A referida consulta foi compreendida também como uma atividade focada na prevenção e encaminhamentos dos problemas, desconsiderando a capacidade resolutiva da consulta de enfermagem e da prática da enfermeira na ESF.

Na parte de prevenção eu me sinto bem confortável, mas lógico que a gente não atende apenas mulheres com condições de saúde perfeitas, mas a gente identifica problemas e encaminha. (E8)

[...] a gente enquanto enfermagem é treinada a identificar alterações, mas a gente não tem, pelo Código de Ética, poder de intervenção nos problemas. (E6)

Observou-se que nenhum dos profissionais entrevistados referiu-se ao protagonismo da enfermagem neste processo, sendo que E6 e E8 parecem desconhecer a capacidade de intervenção da enfermeira sobre os problemas identificados na consulta de enfermagem e da Legislação que as amparam ${ }^{12,13}$.

O conteúdo das falas direciona também para um entendimento de que a resolutividade da APS é deslegitimada, uma vez que a usuária precisará ser encaminhada para um outro serviço, mesmo quando o seu problema poderia ser resolvido localmente. Entretanto, a prática da enfermeira na APS vem evoluindo ao longo dos anos, principalmente na ESF. Este profissional deixou de conduzir apenas funções administradas e passou a desenvolver práticas clínicas diretas com o usuário justamente com o objetivo de ampliar a resolutividade deste nível de atenção9.10.

A consulta de enfermagem consiste em uma atividade a ser desenvolvida exclusivamente pelo profissional enfermeiro, que deve planejá-la de maneira sistemática ancorada na metodologia científica da assistência de enfermagem, proporcionando autonomia profissional. A consulta deve valorizar o acolhimento e ser fundamentada nos princípios da universalidade, integralidade, equidade e resolutividade ${ }^{8,9}$.

É importante ressaltar que na $A B$ a consulta de enfermagem ginecológica apresenta grande potencialidade, pois além da resolução dos problemas clínicos, é uma oportunidade para intervenção nos determinantes sociais. Apesar da demonstração do desconhecimento do fazer profissional por algumas entrevistadas, a Legislação confere legitimidade à atuação do enfermeiro na prescrição de medicamentos estabelecidos em programas de saúde pública e em rotina aprovada pela instituição de saúde $\mathrm{e}^{\not \underline{8}}$.

Portanto, a consulta de enfermagem ginecológica é de grande importância para o cuidado à saúde da mulher, devendo estimular o seu autocuidado, valorizar sua autoestima, orientar a prevenção, controle e tratamento de doenças. É importante também para a enfermagem porque promove a autonomia profissional da enfermeira, troca de saberes entre ela e a mulher atendida, o que favorece uma visão global da saúde da usuária e melhor entendimento das suas necessidades para subsidiar o planejamento do cuidado, considerando a singularidade de cada pessoa atendida. E, para o Serviço, é uma atividade promotora do estreitamento de vínculo, de intervenções numa perspectiva multidisciplinar, integral, colaborativa e mais resolutiva dos problemas de saúde identificados. 
No que tange aos aspectos relativos à saúde da muIher abordados na consulta, observa-se nas falas dos profissionais entrevistados uma tentativa de expandir o olhar para usuária durante o atendimento, contudo, a anamnese tem propósito predominantemente ginecológico e obstétrico.

Quantas gravidezes, se ela quer engravidar, se já engravidou, se ela tá querendo planejamento familiar, informações sobre IST, queixas e condutas, se for preventivo a gente realiza. (E9)

Eu pego menina muito nova, que às vezes teve uma relação sexual e a mãe já acha que tem que fazer

(consulta). (E10)

Nesta fala, fica evidente que a prioridade da consulta ainda é a coleta citopatológica; no entanto, a consulta ginecológica com a enfermeira, muitas vezes, é o único contato da mulher com o serviço de saúde e deve ser vista como uma oportunidade para estreitar a relação entre usuária e profissional, favorecendo uma avaliação da condição biopsicossocial singular da mulher, além de resolver os problemas relatados e necessidades identificadas.

Mudar o paradigma reducionista das consultas ginecológicas não é tarefa fácil. Nesse sentido, a enfermagem entra como protagonista nesse desafio, pois, na maioria das unidades de saúde, esses atendimentos são realizados pela enfermeira. Nesse sentido, a literatura científica destaca a necessidade da valorização do encontro com a usuária, o que potencializará o entendimento e, consequentemente, a redução dos impactos sociais na sua saúde ${ }^{8,14}$.

Uma pesquisa mostrou que as mulheres ouvidas no estudo relataram que se sentiam à vontade nas consultas, pois o profissional as interrogava sobre outros aspectos, como: alimentação, peso, família, o que diminuía, inclusive, a ansiedade na realização da coleta citopatológica ${ }^{15}$.

Outro estudo realizado em Fortaleza constatou que o acolhimento é uma ferramenta extremamente valorizada pelas usuárias nesses momentos, devendo ser priorizada nos atendimentos. Além disso, postura acolhedora favorece o vínculo com a usuária e permite que a enfermeira consiga solucionar problemas que vão além do simples fazer técnico ${ }^{\mathrm{Z}, 14}$.
Ressalta-se que é importante que o profissional conheça "idade, renda, religião, naturalidade, profissão, sexarca, menarca, tipos de parcerias sexuais, frequência das atividades sexuais, uso ou não dos métodos contraceptivos, redes sociais e familiares da mulher, dentre outros" por interferirem diretamente no processo saúde-doença. Os aspectos de raça, gênero e orientação sexual também devem ser considerados, pois existem doenças mais prevalentes em determinadas raças. No caso de muIheres lésbicas ou transexuais, conduzir a consulta de forma adequada, deixando de lado estereótipos heteronormativos que podem afastar essas mulheres do serviço de saúde. Além disso, o ser feminino ainda está relacionado à submissão e, muitas vezes, violência, ressaltando a necessidade do olhar atento neste sentido. Muitas vezes, essa violência influencia na negociação do uso do preservativo, fazendo com que as mulheres se relacionem sem esta proteção, o que as deixa mais vulneráveis às infecções sexualmente transmissíveis (IST) ${ }^{14}$.

É necessário repensar a forma como a consulta ginecológica é vista na ESF, pois o olhar profissional voltado apenas para a busca de fatores de risco gineco-obstétricos impede uma atuação mais contundente nos determinantes sociais da saúde, fazendo com que a prática retorne à lógica ambulatorial fragmentada.

\section{O processo de trabalho de enfermeiras na con- sulta ginecológica de enfermagem}

O trabalho tem um importante papel na evolução do homem, estando na centralidade da vida humana, dando sentido à vida, transformando a sociedade e o humano. Entretanto, o modo capitalista de produção apoia-se na divisão técnica e social do trabalho, comprometendo o caráter humanizador do trabalho 4 .

Através dos depoimentos dos participantes dessa pesquisa foi possível compreender como se dá o planejamento das consultas:

Geralmente é um turno de atendimento todas as quartas-feiras. A gente atende dez por turno. Dez atendimentos são pesados. A gente consegue fazer, mas se fosse menos aumentaria a qualidade. (E10) 
Portanto, cada enfermeira deve disponibilizar uma vez por semana as consultas ginecológicas a serem realizadas em um turno de trabalho. Os profissionais têm autonomia para gerenciar o número de turnos de atendimentos para acolherem a demanda do território, no entanto, a orientação da gestão central e distrital é que em cada turno sejam atendidas dez usuárias. Desse modo, quando o consultório de uma das enfermeiras não se encontra devidamente equipado para a realização ou está interditado devido a qualquer problema, o planejamento é feito para que haja troca de sala entre colegas.

Foi relatado também que a necessidade de organizar a sala antes e após o atendimento acaba subtraindo parte do tempo da consulta, reduzindo ainda mais o contato com a usuária e a escuta qualificada, tornando a consulta mais restrita e menos resolutiva. Isso acaba por favorecer que as consultas de enfermagem tenham caráter meramente prescritivo, limitada à queixa, não levando em consideração a necessidade de estabelecimento de vínculo. Orientações importantes também podem deixar de serem compartilhadas neste processo, conforme também apontaram os estudos de Silva, Gitsos, e Santos e Oliveira ${ }^{8,15}$. As lacunas no atendimento e a carência de informações podem levar as mulheres a buscarem supri-las na internet, o que pode estimular a automedicação e práticas ainda não respaldadas cientificamente.

Ainda é predominante os modelos de gestão ancorados na racionalidade técnico-burocrática, com direcionamentos tempo-espaciais, regulamentado através de resoluções e protocolos que orientam o trabalho em saúde, a partir de deliberações verticalizadas a serem cumpridas pelos profissionais que estão imersos nos cenários da prática do cuidado ${ }^{15}$.

Os participantes falaram também sobre as dificuldades que enfrentam em seu processo de trabalho cotidiano que perpassam também pela consulta ginecológica. As dificuldades mais relatadas por eles foram a ausência de capacitações/orientações a respeito de conteúdo teóricos e práticos relativos à saúde da muIher, bem como dificuldades estruturais e de recursos materiais que levam o profissional a lançar mão de improvisos que acabam por precarizar o atendimento a ser prestado. Dessa forma, alguns profissionais buscam suprir suas carências teóricas e técnicas por iniciativa e custo próprios, como aponta a E4, ou permanecem desatualizados, não acompanhando de forma efetiva os avanços oferecidos na área.

Eu sinto que a gente tem que se aprimorar por conta própria. (E4)

A gente tem muita atualização em vacina, até mesmo pelas mudanças, mas nas outras partes não tem, você fica por fora das atualizações. (E11)

Tem que ter pelo menos uma consulta (ginecológica) anual ou se tiver duas consultas consecutivas, de dois em dois anos, mas já me disseram que isso já mudou

[...]. Várias coisas mudam e a gente pega nos grupos (gestos com a mão apontando para o celular). Sempre acontece alguma coisa, mas na parte de saúde da mulher nunca acontece. (E10)

A ausência de Educação Permanente regular pode gerar heterogeneidade de ações, inclusive entre profissionais atuantes na mesma USF que realizam o cuidado de formas distintas. Assim, a falta de investimento da gestão na qualificação de seus enfermeiros no que diz respeito à consulta ginecológica pode impactar na oferta da atenção necessária à população feminina ou implicar em abordagens inadequadas às usuárias.

O desconhecimento da competência profissional evidencia, ainda mais, a necessidade da educação permanente para qualificar a atuação das enfermeiras e aumentar a resolutividade da APS. É importante ressaltar que, nessa pesquisa, contraditoriamente, os participantes com maior experiência na ESF apresentaram discursos mais incoerentes quando comparados com os que tinham menor tempo de atuação profissional. Tal resultado pode sugerir que a permanência no serviço durante anos, sem atualizações, pode resultar em declínio da competência profissional.

As lacunas existentes no processo de Educação Permanente dos profissionais entrevistados fragilizam o cuidado por eles prestados por gerar insegurança quanto à prescrição de enfermagem, que faz parte da rotina diária desses profissionais:

Enfermeiro tem a autonomia pela abordagem sindrômica de medicar alguma coisa, mas eu não medico via oral. Se precisar via oral eu encaminho para

o Médico. (E5). 
Quando um profissional de saúde não faz uso da autonomia e saber conferida à sua profissão pode comprometer a resolutividade da atenção à saúde, o vínculo da usuária com a USF, o controle de doenças, principalmente as transmissíveis.

No que diz respeito à estrutura física dos consultórios para os atendimentos, as dificuldades foram ainda mais evidentes nos discursos dos entrevistados.

Na realidade nós não temos salas equipadas de forma adequada, no sentido que nem todos os consultórios tem sanitário, aí a gente tem que fazer um rodízio com as equipes, o que é um transtorno ficar mudando de sala. (E6)

São quatro consultórios e 8 profissionais, médicos e enfermeiros dividem a sala, sendo que a agenda precisa ser modificada para que a gente atenda. (E7)

A existência de salas equipadas com banheiro, maca, foco e insumos essenciais é fundamental para o desenvolvimento de uma consulta adequada. A necessidade de improviso gera desmotivação do profissional, uma vez que exigirá mudança de consultório, compreensão de outros profissionais para disponibilizar a sala, transferência de materiais de consumo usuais na consulta e readaptação ao novo ambiente.

Às vezes, quando a demanda está muito alta, você quer estender e colocar mais um turno e acaba ficando sem poder por conta da estrutura da unidade, já que outro profissional também vai precisar. (E8)

Falta de ar-condicionado na sala, insumos materiais, luz que queima, o banheiro vive quebrado, ofoco também que quebra e, às vezes, não resolve logo. (E3)

Observa-se que os pesquisados utilizam manobras e acertos combinados entre eles para operarem o cuidado em saúde. Assim, normas podem ser flexibilizadas e cada profissional acaba adotando um jeito próprio para assegurar a realização do seu trabalho. Nesse sentido, cotidianamente nos serviços de saúde o trabalho prescrito e o trabalho real se confrontam, onde o primeiro se refere às normas, tempo e ferramentas, já o segundo envolve a realidade, muitas vezes distante do ideal16,17.

Fatos podem acontecer de maneira imprevista, o que impossibilita a realização do trabalho prescritivo, padronizado, e o trabalho real acontece em sua substituição. E para que isto aconteça de fato é imprescindível uma relação colaborativa entre os profissionais e compreensão por parte do usuário, que também se insere no espaço do cuidado. Nesse sentido, o processo de trabalho em saúde depende da inter-relação entre sujeitos para que as ações de saúde se efetivem, já que um único profissional não será capaz de sozinho implementar as ações necessárias.

Assim, o vínculo do profissional com o usuário aparece no relato dos entrevistados como o único elemento envolvido no processo que atenua os problemas enfrentados.

A população que é bastante receptiva, o vínculo com a comunidade [...] e aqui os próprios pacientes fazem a sua propaganda. (E11)

O estabelecimento de vínculo entre esses dois atores foi o fator destacado como essencial para motivação profissional, no entanto, o excesso de demanda de trabalho da enfermeira na USF é visto como um dificultador, já que contribui para a redução do contato com cada usuária. Considerando a importância da inter-relação entre profissionais de saúde e usuários, qualquer contexto que restrinja ou tente desqualificar essa relação reverbera na construção de vínculo e na co-responsabilização pela produção de saúde.

\section{Conclusão}

Os resultados deste estudo mostram que a consulta de enfermagem ginecológica faz parte das ações integrantes do processo de trabalho dos profissionais das Unidades Saúde da Família participantes da pesquisa. Revelaram também que as enfermeiras reconhecem a importância, contudo não visualizam o protagonismo da enfermagem na APS.

Embora os relatos tenham expressado um olhar amplo para a saúde da mulher, quando descreviam a prática, evidenciavam atendimentos focados nos aspectos puramente ginecológicos e obstétricos. É importante ressaltar que a enfermeira além de resolver os problemas relatados e necessidades identificadas, deve valorizar a singularidade e o bem-estar biopsicossocial da mulher. 
Foram apontadas dificuldades que perpassam por aspectos normativos, formativos e estruturais, bem como de sobrecarga de trabalho devido ao número de consultas a serem realizadas em um turno de trabalho além das demais ações de sua competência profissional. Contudo, o vínculo que o profissional consegue estabelecer com as usuárias foi considerado o principal elemento motivador para o enfrentamento das dificuldades existentes no processo de trabalho.

Considerando os dados encontrados neste estudo sobre o processo de trabalho da enfermeira na consulta ginecológica no contexto dos serviços de saúde, é possível inferir que nem sempre o trabaIho realizado pela enfermeira será um ato cuidador, uma vez que vários fatores interferem no fazer, bem como a usuária também pode não se sentir cuidada, principalmente quando suas expectativas do cuidado não são atendidas.

O presente estudo apresenta como limitação o fato de ter sido desenvolvido em apenas um DS, não podendo, portanto, indicar a situação real das consultas ginecológicas desenvolvidas por enfermeiras em outros locais. Assim, para as pesquisas futuras, sugerese ampliar o escopo de análise, de modo a mensurar o impacto desses atendimentos restritivos na saúde das mulheres e na resolutividade da APS.

Espera-se que os resultados desse estudo possam contribuir com as reflexões sobre assistência ofertada por enfermeiras na consulta ginecológica, de modo a sensibilizar os profissionais de saúde e gestores para as dificuldades ainda existentes. Pretendese, ainda, contribuir com a valorização da enfermagem e seu protagonismo nesses atendimentos para uma APS mais resolutiva e transformadora.

\section{Contribuições dos autores}

Ribeiro LL participou da concepção, coleta, análise, interpretação dos dados e redação. Góes ACF participou do desenho do estudo, interpretação dos dados, revisão crítica do manuscrito e aprovação da versão final do artigo científico.

\section{Conflitos de interesses}

Nenhum conflito financeiro, legal ou político envolvendo terceiros (governo, empresas e fundações privadas, etc.) foi declarado para nenhum aspecto do trabalho submetido (incluindo, mas não se limitando a subvenções e financiamentos, participação em conselho consultivo, desenho de estudo, preparação de manuscrito, análise estatística, etc.).

\section{Referências}

1. Ministério da Saúde (Brasil), Departamento de Atenção Básica. Controle dos cânceres do colo do útero e da mama [Internet]. 2. ed. Brasília (DF); 2013. Disponível em: https://bvsms.saude.gov.br/ bvs/publicacoes/controle_canceres_colo_utero_2013.pdf

2. Instituto Nacional de Câncer José Alencar Gomes da Silva (Brasil). Diretrizes brasileiras para o rastreamento do câncer do colo do útero [Internet]. 2. ed. Rio de Janeiro (RJ): Ministério da Saúde; 2016. Disponível em: http://www.citologiaclinica.org.br/ site/pdf/documentos/diretrizes-para-o-rastreamento-do-cancerdo-colo-do-utero 2016.pdf

3. Portaria no 2.436, de 21 de setembro de 2017 (Brasil). Aprova a Política Nacional de Atenção Básica, estabelecendo a revisão de diretrizes para a organização da Atenção Básica, no âmbito do Sistema Único de Saúde (SUS) [Internet]. Diário Oficial da União. 2017. Disponível em: https://bvsms.saude.gov.br/bvs/saudelegis/ gm/2017/prt2436_22_09_2017.html

4. Sana MC. Os processos de trabalho em Enfermagem. Rev bras enferm. 2007;60(2): 221-4. https://doi.org/10.1590/S0034$\underline{71672007000200018}$

5. Ministério da Saúde (Brasil), Departamento de ações programáticas estratégicas. Política nacional de atenção integral à saúde da mulher: princípios e diretrizes [Internet]. Brasília (DF): Ministério da Saúde; 2011. Disponível em: https://bvsms.saude. gov.br/bvs/publicacoes/politica_nac_atencao_mulher.pdf

6. Santos MRR. Consulta ginecológica: o que os serviços oferecem e o que as mulheres procuram [dissertação] [Internet]. Porto Alegre: Universidade Federal do Rio Grande do Sul; 2014. Disponível em: https://lume.ufrgs.br/handle/10183/112018

7. Teixeira EHM, Queiroz ABA, Mota MCS, Carvalho MCMP, Costa EPS. Saúde da mulher na perspectiva a assistência prestada pela enfermagem ginecológica: um relato de experiência. Caderno Espaço Fem [Internet]. 2013;26(1):179-89. Disponível em: http:// www.seer.ufu.br/index.php/neguem/article/view/14959 
8. Silva MM, Gitsos J, Santos NLP. Atenção básica em saúde: prevenção do câncer de colo do útero na consulta de enfermagem. Rev Enfer UERJ [Internet]. 2014;21(5):631-6. Disponível em: https://www.e-publicacoes.uerj.br/index.php/ enfermagemuerj/article/view/10039

9. Frigo JÁ, Oliveira DLLC, Rodrigues RM, Zocche DAA. Consulta ginecológica e seu potencial para produzir a integralidade da atenção em saúde. Rev enferm UFPE online [Internet]. 2016;10(4):1299-306. Disponível em: https://periodicos.ufpe.br/ revistas/revistaenfermagem/article/download/11117/12595

10. Catafesta G, Klein DP, Silva EF, Canever BP, Lazzari DD. Consulta de Enfermagem Ginecológica na Estratégia Saúde da Família. Arq Ciênc Saúde. 2015;22(1):85-90. https://doi. org/10.17696/2318-3691.22.1.2015.32

11. Mozzato AR, Grzybovski D. Análise de conteúdo como técnica de análise de dados qualitativos no campo da administração: potencial e desafios. Rev. adm. contemp. 2011;15(4):731-47. https://doi.org/10.1590/S1415-65552011000400010

12. Conselho Federal de Enfermagem. Lei 7.498 de 25 de junho de 1986. Dispõe sobre a regulamentação do exercício da enfermagem, e dá outras providências [Internet]. Diário oficial da União. 1986. Disponível em: http://www.cofen.gov.br/lei-n749886-de-25-de-junho-de-1986 4161.html
13. Decreto no 94.406, de 8 de junho de 1987 (Brasil). Regulamenta a Lei $n^{\circ} 7.498$, de 25 de junho de 1986, que dispõe sobre o exercício da Enfermagem, e dá outras providências [Internet]. Diário oficial da União. 1986. Disponível em: http:// www.planalto.gov.br/ccivil_03/decreto/1980-1989/d94406. htm\#: :text=Regulamenta\%20a\%20Lei\%20n\%C2\%BA\%20 7.498,enfermagem $\% 2$ C $\% 20 \mathrm{e} \% 20 \mathrm{~d} \% \mathrm{C} 3 \% \mathrm{~A} 1 \% 20$ outras $\% 20$ provid\%C3\%AAncias

14. Pereira SM, Taquette SR. La consulta ginecológica en la adolescência bajo la optica de médicos ginecólogos de la ciudad de Rio de Janeiro. Rev chil. Obstet. Ginecol. 2011;76(5):311-7. http://dx.doi.org/10.4067/S0717-75262011000500005

15. Oliveira SKP, Queiroz APO, Matos DPM, Moura AF, Lima FET. Temas abordados na consulta de enfermagem: revisão integrativa da literatura. Rev Bras de Enf. 2012;65(1):155-61. https://doi. org/10.1590/S0034-71672012000100023

16. Farias HX, Araújo MD. Uma Perspectiva de Análise sobre o Processo de Trabalho em Saúde: produção do cuidado e produção de sujeitos. Saude Soc. 2010;19(2):429-39. http://dx.doi. org/10.1590/S0104-12902010000200018

17. Merhy EE. Saúde: cartografia do trabalho vivo. São Paulo: Hucitec; 2007. 